\title{
Sponsoring Content: Motivation and Pitfalls for Content Service Providers
}

\author{
Liang Zhang*, Dan Wang*‡ \\ * Department of Computing, The Hong Kong Polytechnic University \\ $\ddagger$ The Hong Kong Polytechnic University Shenzhen Research Institute \\ Email: \{cslizhang, csdwang\}@comp.polyu.edu.hk
}

\begin{abstract}
With the popularity of bandwidth-intensive mobile applications and mobile devices, the data traffic is increasing fast nowadays. This poses huge burden to the Internet service providers (ISPs) to support such wireless data traffic as they need to invest on developing advance networks, e.g., 4G, or expending the capacity of the current networks in a much faster pace. One way to keep up financing such investment is to transfer the costs to the end users. There are studies on new payment models, e.g., time-dependent pricing. An alternative way is sponsored content. More specifically, the content service providers (CSPs) can sponsor the end users for the traffic of viewing their content. As an example, Google, with India ISPs, is currently sponsoring Gmail, Google+, etc. for its end users.
\end{abstract}

Nevertheless, much is unknown about the impact of such strategy on the CSPs of different scales, more specifically, whether richer CSPs may harvest more advantage on the competitive edge. In this paper, we first analyze the interplay among CSPs, a monopolistic ISP and end users. We conclude: 1) small CSPs and large (or rich) CSPs all may have part incentive to sponsor content for its end users when the monopolistic ISP cannot discriminate the charging price of CSPs; and 2) otherwise, none of them has the incentive to adopt this strategy. We then study the effect of competition from short-run (i.e., market shares are fixed) and long-run (i.e., market shares are dynamic) perspectives in the market with one small CSP and one large CSP. We show that the small CSP (or large CSP) may benefit more from the adoption of sponsored content for the short-run (or long-run) competition.

\section{INTRODUCTION}

With the popularity of bandwidth-intensive mobile devices such as smart phones, tablet computers, etc., the data traffic is increasing fast nowadays. This poses huge burden to the Internet service providers (ISPs) to support such wireless data traffic. The ISPs always supply traffic plans with low data caps due to the scarcity of bandwidth. The pricing data set revealed by Google demonstrates that almost $85 \%$ of all plans offer less than $10 \mathrm{~GB}$ of data a month, and $36 \%$ offer less than $1 \mathrm{~GB}$ a month [1]. Even for $10 \mathrm{~GB}$ monthly data plan, it would be fully exhausted in less than seven hours under current 3G bandwidth [2]. Meeting this demand-supply gap requires large investment by building advanced networks, e.g., 4G, or extending the capacity of the current networks with more bandwidths and base stations. One way to keep up financing such investment is to transfer the costs to the end users. There are extensively studies on new payment models, e.g., timedependent pricing [3], [4].

An alternative way is sponsored content. More specifically, the content service providers (CSPs) ${ }^{1}$ can sponsor the end users by direct payments to ISPs or sharing advertising revenue with them. When end users access the content provided by the CSPs, their traffic is partly or fully exempted from their mobile data caps. For example, Google has joined with India's Bharti Airtel to offer free access to certain Google-based services such as Gmail, Google+ and first page of web sites via Google search without ringing up data charges [5]. This pricing strategy creates a positive cycle: end users are glad to access more content without counted into their mobile data cap; CSPs can attract more users and views; ISP can get more revenue by this new revenue resource so as to support better performance and technology update. It also removes the inefficiency of current arrangement under which the CSPs derive profit from showing advertisements while end users pay the cost of viewing them.

Nevertheless, this new pricing model in cellular networks has been linked to net neutrality debate recently, because some content wouldn't be counted against monthly data caps set by CSPs [6]. The net neutrality advocators, including representatives from public interests group, concern that such plan will favor rich and large CSPs over small ones [7]. In fact, net neutrality rules have been heavily discussed from the points of computer science, economics and law. Yet, the core issue of net neutrality for this new pricing model is whether the CSPs will compete unfairly under this policy, instead of the traditional issues such as whether the ISPs can transmit packets with priority or the last-mile ISPs can charge CSPs for the content transmitting to their consumers. The CSPs also change their aptitude to network neutrality from showing strong proponent to being interested in this new pricing model [8]. For example, ESPN tried to talk with US's mobile carriers about the toll-free data plans [9]. It remains an interesting question: which kind of CSPs will prefer this new pricing strategy. The concern announced by the neutrality advocators is also deserved detailed discussion.

In this paper, we focus on the impact of sponsored content strategy on the CSPs of different scales. We first provide an analysis on the complicated interplay among CSPs, the monopolistic ISP and end users. Then, we consider the effect of competition for the adoption of sponsored content policy from short-run (i.e., market shares are fixed) and long-run (i.e., market shares are dynamic) perspectives in the market with one small CSP and one large CSP. Our study shows the following conclusions:

\footnotetext{
${ }^{1}$ Content service provider is also known as Internet content provider (ICP).
} 
- When the monopolistic ISP cannot discriminate the charging price of CSPs, small CSPs and large CSPs all may have part incentive to sponsor content. Otherwise, none of them adopts sponsored content plans.

- $\quad$ For the short-run competition, the small CSP is more likely to benefit from sponsored content plans but not for the large one.

- For the long-run competition, the market share of the large CSP becomes larger and the small CSP becomes smaller.

\section{RELATED WORK}

There are extensive studies on network neutrality problem despite of a short history. Ma et al. [10] focused on paid prioritization of content analyzed from the view of consumers. They introduced the notation of public option ISP showed to be a better choice than network neutral manner for both monopolistic ISP and oligopolistic scenario. Hande et al. [11] studied the strategy of pricing content-providers for their connectivity to end-users. They showed that, in addition to gains in total and end-user surplus, content-providers will experience a net surplus from participation in rate allocation under low cost of connectivity. Choi et al. [12] studied the effect of neutral regulations on investment incentives for ISPs and CSPs. They found that capacity expansion decreases the sale price of the premium service.

Nevertheless, none of them considered the sponsored content strategy. In fact, there are currently few academic works directly related to this new pricing strategy. From the economic point of view, Andrews et al. [13] studied the contractual relationship between the service providers and content providers. They modeled the interaction between the service providers and the content providers as a Stackelberg game with random demand. Their work concluded that a coordinating contract can maximize total system profit and the additional profit. From the technical point of view, Raj et al. [2] developed a new computing abstraction, called SIMlet, based on the idea of split billing. The SIMlet can provide trustworthy proofs of a device's mobile traffic that can be redeemed at content provider involved in split billing. Other studies, e.g., timedependent pricing (TDP), are orthogonal to sponsored content strategy. For example, Ha et al. [3] presented the architecture, implementation, and a user trial of the system for TDP, called TUBE. We also have a theoretical study [14], [15] about the incentives of TDP and comparison between usage-based and flat-rate schemes in TDP.

In this paper, we focus on the impact of sponsored content strategy on the CSPs of different scales. We demonstrate which kind of CSPs prefer the sponsored content strategy and whether this new pricing strategy will result in unfair competition between rich and large CSPs and small CSPs.

\section{BASIC SYSTEM MODEL}

In this section, we consider the basic system model in the market with CSPs, a monopolistic ISP and end users. The monopolistic ISP provides the connection services to CSPs and end users. The users view the content provided by CSPs. We denote $x$ as the traffic volume of a CSP viewed by end users.
Let $\sigma_{e} f(x)$ be the utility obtained from viewing the content of the CSP, where factor $\sigma_{e}$ represents the utility level of end users. We assume that $f(\cdot)$ is a non-decreasing and concave function with decreasing marginal satisfaction. This reflects the decreasing marginal preference of end users. The ISP charges end users $p_{e}$ for per unit traffic. ${ }^{2}$ This price can be partly sponsored by the CSP. We denote the sponsored percentage as $\alpha$. Thus, end users can decide their optimal traffic volume by maximizing their own payoff as follows:

$$
\begin{array}{rl}
\text { (EU-P): } \max _{x} & u(x)=\sigma_{e} f(x)-(1-\alpha) x p_{e} \\
\text { s.t. } & x \geq 0 .
\end{array}
$$

Given the price charged by the ISP and the sponsored percentage, i.e., $\left\{p_{e}, \alpha\right\}$, the optimal traffic usage for end users is:

$$
x_{e}^{*}=f^{\prime-1}\left((1-\alpha) p_{e} / \sigma_{e}\right),
$$

where $f^{\prime-1}(\cdot)$ is the inverse function of first order derivative of $f(\cdot)$. A common example of function $f(\cdot)$ is $f(x)=$ $\frac{1}{1-\beta_{e}} x^{1-\beta_{e}}, 0<\beta_{e}<1$. In this case, the optimal traffic consumption is $x_{e}^{*}\left(\alpha, p_{e}\right)=\left(\frac{\sigma_{e}}{(1-\alpha) p_{e}}\right)^{1 / \beta_{e}}$. The price elasticity can be expressed as $\epsilon_{e}=-\frac{\partial x^{*}\left(\alpha, p_{e}\right)}{\partial p_{e}} \frac{p_{e}}{x^{*}\left(\alpha, p_{e}\right)}=\frac{1}{\beta_{e}}$, which characterizes how much the rate provisioning changes as the price changes. Small price elasticity means that it is harder to change the traffic consumption by charging price $p_{e}$.

Similarly, we consider the payoff of CSPs. We use $\sigma_{e} g(x)$ to represent the revenue of the CSP, where $\sigma_{e}$ reflects the revenue level of the CSP. Different types of CSPs may have much different revenue levels [16]. Even for the same type of CSPs, the revenue levels may be much different. For instance, the large CSPs usually have higher revenue level than that of small ones [17]. The revenue can be generated by advertisement, e.g., YouTube, or by value-added services, e.g., Tencent, and other e-commerce, e.g., Amazon. We assume that $g(\cdot)$ is a non-decreasing and concave function. It means that the marginal revenue decreases with the increase of traffic. The monopolistic ISP charges CSPs $p_{c}$ for per unit traffic. The payoff function for this CSP is:

$$
v(x)=\sigma_{c} g(x)-\alpha x p_{e}-x p_{c} .
$$

Given the sponsored percentage $\alpha$, the price per unit charged to CSPs $p_{c}$ and end users $p_{e}$, the CSP also has its own optimal traffic volume:

$$
x_{c}^{*}=g^{\prime-1}\left(\left(\alpha p_{e}+p_{c}\right) / \sigma_{c}\right) .
$$

With the function $g(x)=\frac{1}{1-\beta_{c}} x^{1-\beta_{c}}, 0<\beta_{c}<1$, the optimal traffic volume for the CSP is $x_{c}^{*}\left(\alpha, p_{e}, p_{c}\right)=\left(\frac{\sigma_{c}}{\alpha p_{e}+p_{c}}\right)^{1 / \beta_{c}}$. The price elasticity of the CSP is $\epsilon_{c}=-\frac{\partial x_{c}^{*}\left(\alpha, p_{c}\right)}{\partial p_{c}} \frac{p_{c}}{x_{c}^{*}\left(\alpha, p_{c}\right)}=$ $\frac{1}{\beta_{c}}$. Note that the effective traffic volume is decided by both end users and the CSP, i.e., $x^{*}=\min \left\{x_{c}^{*}, x_{e}^{*}\right\}$. If $x_{c}^{*}<x_{e}^{*}$, then the effective traffic volume is limited by the CSP. For example, some web sites limit the number of simultaneous online users due to the gap between limited capacity of servers and huge demand. If $x_{c}^{*} \geq x_{e}^{*}$, then the effective traffic volume is just

\footnotetext{
${ }^{2}$ In practice, most of current plans provided by ISPs are in form of fixed prices for some data caps, instead of usage-based prices. Yet, this form of pricing strategy can be viewed as usage-based pricing strategy since both schemes encourage users viewing more important content [14].
} 
end users' demand. The CSP decides the optimal sponsored percentage with this effective volume by solving the following problem:

$$
\begin{array}{cl}
\text { (CSP-P): } \max _{\alpha} & v(\alpha)=\sigma_{c} g\left(x^{*}(\alpha)\right)-\alpha x^{*}(\alpha) p_{e}-x^{*}(\alpha) p_{c} \\
\text { s.t. } & 0 \leq \alpha \leq 1 .
\end{array}
$$

Since $v(\alpha)$ is continuous and bounded over interval $[0,1]$, the optimal solution of above optimization exists. In general, it is hard to quantify the properties of this optimal solution. In order to show some interesting insights, we consider the special case where $\beta_{e}=\beta_{c}=\beta$. When $\alpha \leq \frac{\sigma_{c} p_{e}-\sigma_{e} p_{c}}{\left(\sigma_{e}+\sigma_{c}\right) p_{e}}$, the effective traffic volume is decided by traffic usage of end usage, i.e., $x^{*}=x_{e}^{*}$. Similarly, when $\alpha \geq \max \left\{\frac{\sigma_{c} p_{e}-\sigma_{e} p_{c}}{\left(\sigma_{e}+\sigma_{c}\right) p_{e}}, 0\right\}$, the effective traffic volume is decided by best traffic volume of the CSP, i.e., $x^{*}=x_{c}^{*}$. In particular, when $\frac{\sigma_{c}}{\sigma_{e}} \leq \frac{p_{c}}{p_{e}}$, we have $x^{*}=x_{c}^{*}$. The following theorem shows the optimal sponsored percentage of the CSP to end users.

Theorem 1: Given the prices $\left\{p_{c}, p_{e}\right\}$ charged by the ISP to CSPs and end users, the optimal sponsored percentage $\alpha^{*}\left(p_{c}, p_{e}\right)$ for the CSP is:

$$
\alpha^{*}\left(p_{c}, p_{e}\right)= \begin{cases}0 & \text { if } \frac{\sigma_{c}}{\sigma_{e}} \leq \beta+\frac{p_{c}}{p_{e}}, \\ \frac{\sigma_{c} / \sigma_{e}-\beta-p_{c} / p_{e}}{\sigma_{c} / \sigma_{e}+1-\beta} & \text { if } \frac{\sigma_{c}}{\sigma_{e}}>\beta+\frac{p_{c}}{p_{e}} .\end{cases}
$$

Due to the limit of space, all proofs in this paper are omitted and available in the technical report [18].

Theorem 1 states that the CSP supplies no sponsored content to its end users, i.e., $\alpha^{*}=0$, when the condition $\frac{\sigma_{c}}{\sigma_{e}} \leq \beta+\frac{p_{c}}{p_{e}}$ is satisfied. Otherwise, the CSP has strong incentive to supply sponsored content plan for its content. In particular, when $\sigma_{c} \gg \sigma_{e}$, the CSP undertakes almost all the traffic cost of its end users generated by viewing its content. In other words, the content provided by the CSP with high revenue level and consumed by end users with relatively low utility level are more likely to be toll-free under the CSP's sponsored content plan. The higher price charged to end users and lower price charged to the CSP results in the higher sponsored percentage. For example, Google has strong incentive to supply sponsored content plan to end users due to two reasons: (1) the revenue levels are much high for its services, such as Gmail and searching service; and (2) the transit costs for Google are close to zero due to its homegrown infrastructure [19], i.e., $\frac{p_{c}}{p_{e}}$ is small.

With the optimal sponsored percentage of content, the effective traffic volume can be determined:

$$
x^{*}\left(p_{c}, p_{e}\right)= \begin{cases}\left(\frac{\sigma_{c}}{p_{c}}\right)^{1 / \beta} & \text { if } \frac{\sigma_{c}}{\sigma_{e}} \leq \frac{p_{c}}{p_{e}} \\ \left(\frac{\sigma_{e}}{p_{e}}\right)^{1 / \beta} & \text { if } \frac{p_{c}}{p_{e}}<\frac{\sigma_{c}}{\sigma_{e}} \leq \beta+\frac{p_{c}}{p_{e}}, \\ \left(\frac{\sigma_{c}+(1-\beta) \sigma_{e}}{p_{c}+p_{e}}\right)^{1 / \beta} & \text { if } \frac{\sigma_{c}}{\sigma_{e}}>\beta+\frac{p_{c}}{p_{e}} .\end{cases}
$$

The effective traffic volume are divided into tree regions, corresponding to tree types of CSPs shown in Fig. 1. The three types of CSPs are as follows:

- Non-incentive CSPs: the CSPs that have lower expected traffic usage than end users. It means that end users are interested in the content provided by nonincentive CSPs, but the CSPs can hardly support such huge amount of traffic due to low revenue level and high cost. Obviously, the CSPs have no incentive to offer sponsored content plan;

- Potential CSPs: the CSPs that have higher willingness to supply content than the demand but do not provide sponsored content plan to end users. The potential CSPs have higher cost of sponsoring the content than the benefit from this strategy;

- Incentive CSPs: the CSPs that have strong incentive to sponsor end users viewing its content. The revenue level of the incentive CSPs is relatively high while the utility level of their end users is relatively low. The proper sponsored content plan can encourage traffic usage of end users and increase the profit of this type of CSPs.

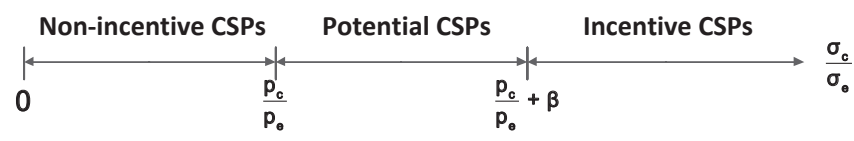

Fig. 1: Three types of CSPs

The monopolistic ISP obtains its revenue from charging CSPs and end users. If the ISP can discriminate the prices of CSPs, it can decide optimal prices $\left\{p_{c}, p_{e}\right\}$ charged to the CSP and end users, respectively, so as to maximize its profit. This profit is impacted by two factors: 1) the service fees charged to the CSP and end users, modeled as $p_{c} x^{*}$ and $p_{e} x^{*}$ respectively; and 2) the variable $\operatorname{cost}^{3}$, modeled as $c x^{*}$, where $c$ is the marginal cost. Thus, the ISP determines the optimal prices $\left\{p_{c}, p_{e}\right\}$ by solving the following problem:

$$
\begin{aligned}
\text { (ISP-P): } \max _{\left\{p_{e}, p_{c}\right\}} & \pi=\left(p_{e}+p_{c}-c\right) x^{*}\left(p_{c}, p_{e}\right) \\
\text { s.t. } & p_{e} \geq 0, p_{c} \geq 0 .
\end{aligned}
$$

Theorem 2: With the best response of the CSP and end users, the optimal prices $\left\{p_{c}^{*}, p_{e}^{*}\right\}$ charged by the monopolistic ISP to the CSP and end users are:

$$
p_{c}^{*}=\frac{c \sigma_{c}}{\left(\sigma_{c}+\sigma_{e}\right)(1-\beta)}, p_{e}^{*}=\frac{c \sigma_{e}}{\left(\sigma_{c}+\sigma_{e}\right)(1-\beta)} .
$$

Theorem 2 shows the best price strategy for the monopolistic ISP. Note that $\frac{p_{c}^{*}}{p_{e}^{*}}=\sigma_{c} / \sigma_{e}$. According to theorem 1 , we have $\alpha^{*}=0$. It means that with the monopolistic power for the ISP to discriminate the prices of CSPs, the best strategy of the CSP is not to adopt the plan of sponsored content. The intuition is that the monopolistic ISP eliminates the gap between the optimal traffic volume for CSPs and traffic demand for end users by pricing them in an optimal way resulting in no incentive for CSPs sponsoring content. Yet, in practice, the monopolistic ISP cannot discriminate the prices of CSPs due to the network neutrality rules. In fact, the price charged to CSPs is usually low. The current CDNs charge the traffic distribution for about $\$ 0.01$ to $\$ 0.02$ per GB [20], much lower than the price charged to end users by ISPs. YouTube even has built its own homegrown infrastructure so as to reduce transit

\footnotetext{
${ }^{3}$ The total cost consists of variable cost and fixed cost. We only consider the variables cost since the fixed cost is just a constant and does not affect the conclusions.
} 
costs [19]. Hence, we consider the case of ignoring the price charged to the CSP, i.e., $p_{c}=0$. The optimal content sharing percentage is demonstrated in the following corollary.

Corollary 1: When $p_{c}=0$, the optimal price charged by the ISP to end users is $p_{e}^{*}=\frac{c}{1-\beta}$. The optimal content sharing percentage $\alpha^{*}$ is:

$$
\alpha^{*}\left(p_{e}\right)= \begin{cases}0 & \text { if } \frac{\sigma_{c}}{\sigma_{e}} \leq \beta \\ \frac{\sigma_{c} / \sigma_{e}-\beta}{\sigma_{c} / \sigma_{e}+1-\beta} & \text { if } \frac{\sigma_{c}}{\sigma_{e}}>\beta .\end{cases}
$$

Corollary 1 demonstrates that the optimal price charged to end users is $\frac{c}{1-\beta}$, much higher than that when the monopolistic ISP can discriminate the prices of CSPs. When the content is sponsored partly by CSPs, i.e., $\frac{\sigma_{c}}{\sigma_{e}}>\beta$, the fee paying to the ISP by end users is still higher. Note that the summation of prices for CSPs and end users in Theorem 2 is equal to the optimal price in corollary 1 . It means that when the monopolistic ISP can discriminate the price of CSPs, it can transfer part of the cost from end users to the CSP. Yet, when the ISP cannot discriminate the price of CSPs, the sponsored content strategy can be still a good choice for the ISP to etransfer its cost to the CSP. Corollary 1 also shows that the sponsored percentage is independent of the price to end users. This percentage only depends on the revenue level of the CSP and its users' utility level.

Summary. When the monopolistic ISP cannot discriminate the prices of CSPs, the CSPs can be divided into three types, i.e., non-incentive CSPs, potential CSPs and incentive CSPs, according to their incentives of adopting sponsored content strategy. This strategy provides an efficient way to transfer the cost of the ISP from end users to CSPs. Otherwise, the monopolistic ISP eliminates the gap between the optimal traffic volume for CSPs and traffic demand for end users by pricing them in an optimal way resulting in no incentive for CSPs sponsoring content.

\section{COMPETITION ANALYSIS}

In this section, we study the effect of competition to the adoption of sponsored content for CSPs. We consider the market with one small CSP and one large CSP. The large CSP refers to the CSP that has high revenue level while the small CSP has low revenue level. In addition, the initial number of end users of the large CSP is also much higher than that of the small one. We divide the competition of two CSPs into two cases: short-run competition and long-run competition. In short-run competition, the numbers of end users for both CSPs are fixed while in the long-run competition the numbers of end users are changed with the time.

\section{A. Short-run competition}

For short-run competition, the number of end users for the two CSPs are assumed to be unchanged due to the consistent usage habits during a short time. We denote $n_{i}, i=1,2$ as the user number of CSP $i$. The number of end users for both CSPs can also be treated as the market share if we normalize total number of end users to 1 . Without loss of generality, we let CSP 1 be the small one and CSP 2 be the large one, i.e., $n_{1} \leq n_{2}$. We assume that the monopolistic ISP cannot charge different CSPs the different prices. We denote the revenue level of CSP $i$ as $\sigma_{c_{i}}$. Note that the revenue level of the large one is higher, i.e., $\sigma_{1} \leq \sigma_{2}$. The utility level for the end users of CSP $i$ is denoted as $\sigma_{e_{i}}$. Either the small CSP or large CSP may have higher utility level for its end users. For instance, the positive network externality own by large CSP or the better services provided by small CSPs can make the end users have high utility level. The percentage of sponsored content for CSP $i$ is denoted as $\alpha_{i}$. Given the price charged by the monopolistic ISP, the optimal reactions for the CSPs and end users are the same with section III. We denoted the effective traffic volume for CSP $i$ as $x_{i}^{*}\left(p_{c}, p_{e}\right)$. Here, we only need consider the optimal price $\left\{p_{c}, p_{e}\right\}$ decided by maximizing the utility function of the ISP:

$$
\begin{aligned}
\text { (ISP-P): } \max _{\left\{p_{e}, p_{c}\right\}} & \pi=\left(p_{e}+p_{c}-c\right)\left(n_{1} x_{1}^{*}\left(p_{c}, p_{e}\right)+n_{2} x_{2}^{*}\left(p_{c}, p_{e}\right)\right) \\
\text { s.t. } & p_{e} \geq 0, p_{c} \geq 0
\end{aligned}
$$

Theorem 3: The optimal prices $\left\{p_{c}, p_{e}\right\}$ charged by the monopolistic ISP are one of the following cases:

$$
\begin{gathered}
p_{c}^{*}=\frac{c \sigma_{c_{1}}}{\left(\sigma_{c_{1}}+\sigma_{e_{1}}\right)(1-\beta)}, p_{e}^{*}=\frac{c \sigma_{e_{1}}}{\left(\sigma_{c_{1}}+\sigma_{e_{1}}\right)(1-\beta)}, \\
p_{c}^{*}=\frac{c \sigma_{c_{2}}}{\left(\sigma_{c_{2}}+\sigma_{e_{2}}\right)(1-\beta)}, p_{e}^{*}=\frac{c \sigma_{e_{2}}}{\left(\sigma_{c_{2}}+\sigma_{e_{2}}\right)(1-\beta)} .
\end{gathered}
$$

Theorem 3 shows that the optimal price is only decided by one CSP. In both cases, the summations of prices for CSPs and end users are the same, i.e., $\frac{c}{1-\beta}$. For the first case, the optimal price only depends on the revenue level of the CSP 1 and the utility of its end users. This happens when the numbers of end users, utility levels and revenue levels for CSP 1 and 2 satisfy the following condition:

$$
\begin{aligned}
& n_{1}+n_{2}\left[\max \left\{\frac{\sigma_{e_{2}}}{\sigma_{e_{1}}}, \frac{\sigma_{c_{2}}+(1-\beta) \sigma_{e_{2}}}{\sigma_{c_{1}}+\sigma_{e_{1}}}\right\}\right]^{1 / \beta} \\
& \geq\left[n_{1}\left(\frac{\sigma_{c_{1}}}{\sigma_{c_{2}}}\right)^{1 / \beta}+n_{2}\right]\left(\frac{\sigma_{c_{2}}+\sigma_{e_{2}}}{\sigma_{c_{1}}+\sigma_{e_{1}}}\right) .
\end{aligned}
$$

Otherwise, the optimal price depends on the CSP 2, i.e., $\frac{p_{c}^{*}}{p_{e}^{*}}=$ $\frac{\sigma_{c_{2}}}{\sigma_{e_{2}}}$. Usually, the number of the large CSP is much higher than the small one. Thus, we have the following proposition.

Proposition 1: When $n_{1} \ll n_{2}$, the percentages of sponsored content for two CSPs are as follows:

$$
\alpha_{1}^{*}= \begin{cases}0 & \text { if } \frac{\sigma_{c_{1}}}{\sigma_{e_{1}}} \leq \beta+\frac{\sigma_{c_{2}}}{\sigma_{e_{2}}} \\ \frac{\sigma_{c_{1}} / \sigma_{e_{1}}-\beta-\sigma_{c_{2}} / \sigma_{e_{2}}}{\sigma_{c_{1}} / \sigma_{e_{1}}-\beta+1} & \text { if } \frac{\sigma_{c_{1}}}{\sigma_{e_{1}}}>\beta+\frac{\sigma_{c_{2}}}{\sigma_{e_{2}}}\end{cases}
$$

and $\alpha_{2}^{*}=0$.

Proposition 1 demonstrates that the large CSP doesn't benefit from the sponsored content strategy. The small CSP benefits from this strategy if and only if the revenue level and the utility level of its end users satisfy $\frac{\sigma_{c_{2}}}{\sigma_{e_{2}}}+\beta \leq \frac{\sigma_{c_{1}}}{\sigma_{e_{1}}}$. The intuition is that the ISP can obtain more profit by charging price closer to the optimal price for the large CSP. This price eliminates the incentive of sponsoring content for large CSP but leaves enough space to the small one. When considering the special case, i.e., $p_{c}=0$, we have the following corollary.

Corollary 2: When $p_{c}=0$, the optimal price charged by the ISP to end users is $p_{e}^{*}=\frac{c}{1-\beta}$. The optimal sponsored percentage for CSP $i$ can be shown as:

$$
\alpha_{i}^{*}\left(p_{c}, p_{e}\right)= \begin{cases}0 & \text { if } \frac{\sigma_{c_{i}}}{\sigma_{e_{i}}} \leq \beta, \\ \frac{\sigma_{c_{i}} / \sigma_{e_{i}}-\beta}{\sigma_{c_{i}} / \sigma_{e_{i}}+1-\beta} & \text { if } \frac{\sigma_{c_{i}}}{\sigma_{e_{i}}}>\beta .\end{cases}
$$


Similar to the corollary 1 , when the ISP can only charge end users, the optimal price is independent of the revenue level of CSPs and the utility level of end users. In addition, the optimal percentage of sponsored content for each CSP is also independent of the number of their end users. In other words, each CSP just decide how much to sponsor its end users according to its own revenue level and the utility level of its end users.

\section{B. Long-run competition}

The market shares for each CSP are usually changed if we consider long-run market with competition. They are greatly affected by the payoff of end users. The CSP with higher payoff of its end users is more likely to have much higher market share. The most commonly used economic model for market shares is Hotelling Model. The Hotelling Model assumes that the end users are uniformly located along a unit interval with two CSPs located at two endpoints. Each user has transportation cost by unit of distance squared, denoted as $t$, when deciding its CSP. If the end user is at a distance $n_{i}$ to one of the CSPs, its transportation cost is $t n_{i}^{2}$. The transportation cost per unit can be much different for different kinds of services. For example, the users of Facebook surfer high transportation cost per unit to other social network services such as Google+ while the users of YouTube can easily visit other video sharing sites if the required videos provided. With the Hotelling Model, given the payoff of end users for CSP $i$, e.g., $u_{i}$, the market share for CSP $i$ is:

$$
n_{i}=\frac{1}{2}+\frac{u_{i}-u_{-i}}{2 t}
$$

where $u_{-i}$ denotes the utility of end users not belonging to CSP $i$. Each CSP competes for the market share by improving the payoff level of its end users, e.g., increasing the sponsored percentage. Meanwhile, it also has to balance the market share and the profit obtained from one end user. The total profit of CSP $i$ is:

$$
v_{i}\left(x_{i}\right)=n_{i} \sigma_{c_{i}} g\left(x_{i}\right)-\left(\alpha_{i} p_{e}+p_{c}\right) n_{i} x_{i} .
$$

Given the percentage of sponsored content $\boldsymbol{\alpha}, \operatorname{CSP} i$ has its own optimal traffic volume. Considering the first-order derivative of the profit of CSP $i$, we have:

$$
\begin{aligned}
\frac{\partial v_{i}\left(x_{i}\right)}{\partial x_{i}}= & \frac{\sigma_{e_{i}} f^{\prime}\left(x_{i}\right)-\left(1-\alpha_{i}\right) p_{e}}{2 t}\left[\sigma_{c_{i}} g\left(x_{i}\right)-\left(\alpha_{i} p_{e}+p_{c}\right) x_{i}\right] \\
& +n_{i}\left(\sigma_{c_{i}} g^{\prime}\left(x_{i}\right)-\alpha_{i} p_{e}-p_{c}\right)=0 .
\end{aligned}
$$

Putting the optimal traffic usage for end users, i.e., $x_{e_{i}}^{*}=$ $\left(\frac{\sigma_{e_{i}}}{\left(1-\alpha_{i}\right) p_{e}}\right)^{1 / \beta}$ into the above one-order derivative of the profit for CSP $i$, it follows:

$$
\left.\frac{\partial v_{i}\left(x_{i}\right)}{\partial x_{i}}\right|_{x_{i}=x_{e_{i}}^{*}}=n_{i}\left[\frac{\sigma_{c_{i}}}{\sigma_{e_{i}}}\left(1-\alpha_{i}\right) p_{e}-\alpha_{i} p_{e}-p_{c}\right] .
$$

When $\alpha_{i} \leq \frac{\sigma_{c_{i}} / \sigma_{e_{i}}-p_{e} / p_{c}}{1+\sigma_{c_{i}} / \sigma_{e_{i}}}$, we have $\left.\frac{\partial v_{i}\left(x_{i}\right)}{\partial x_{i}}\right|_{x_{i}=x_{e_{i}}^{*}} \geq 0$. For another traffic usage $x_{i}=\left(\frac{\sigma_{c_{i}}}{\alpha_{i} p_{e}+p_{c}}\right)^{1 / \beta} \geq x_{e_{i}}^{*}$, we

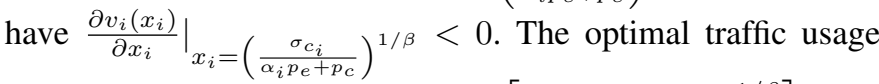
for CSP $i$ is within the interval $\left[x_{e_{i}}^{*},\left(\frac{\sigma_{c_{i}}}{\alpha_{i} p_{e}+p_{c}}\right)^{1 / \beta}\right]$. The optimal traffic usage for CSP $i$, denoted as $x_{c_{i}}^{*}$ is larger

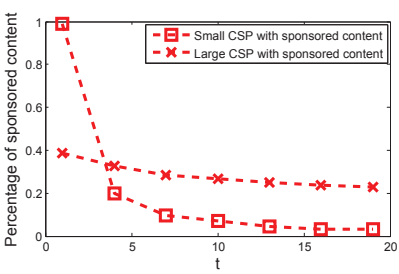

(a) Small CSP with higher user utility level $\left(\sigma_{e_{1}}=1, \sigma_{e_{2}}=1.5\right)$

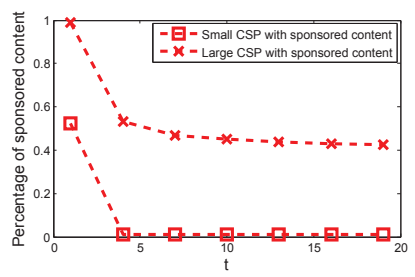

(b) Large CSP with higher user utility level $\left(\sigma_{e_{1}}=1.5, \sigma_{e_{2}}=1\right)$
Fig. 2: An example of the percentage of sponsored content in the long run $\left(\sigma_{c_{1}}=1, \sigma_{c_{2}}=2, \beta=0.5, p_{e}=0.5, p_{c}=1\right)$

than $x_{e_{i}}^{*}$, i.e., the optimal effective traffic usage is $x_{i}^{*}=$ $\left(\frac{\sigma_{e_{i}}}{\left(1-\alpha_{i}\right) p_{e}}\right)^{1 / \beta}$. When $\alpha_{i} \geq \frac{\sigma_{c_{i}} / \sigma_{e_{i}}-p_{e} / p_{c}}{1+\sigma_{c_{i}} / \sigma_{e_{i}}}$, we have $x_{i}=$

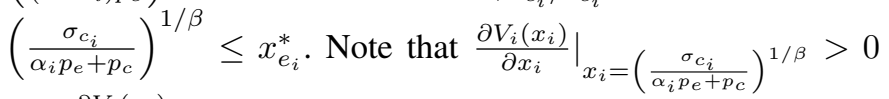
and $\left.\frac{\partial V_{i}\left(x_{i}\right)}{\partial x_{i}}\right|_{x_{i}=x_{e_{i}}^{*}} \leq 0$. The optimal traffic usage for CSP $i$ is within the interval $\left[\left(\frac{\sigma_{c_{i}}}{\alpha_{i} p_{e}+p_{c}}\right)^{1 / \beta}, x_{e_{i}}^{*}\right]$. The effective traffic usage is $x_{i}^{*}=x_{c_{i}}^{*}$. Thus, we can divide the original optimization problem into two, one with effective traffic usage decided by end users and the other decided by the CSP $i$ itself. Given the sponsored percentage $\alpha_{-i}$, where $\alpha_{-i}$ is the percentage of sponsored content for CSPs except $i$, we can get the optimal reaction by analyzing these two optimization problems. Denote $\alpha^{*}$ to be the Nash equilibrium [21] of sponsored percentage. We have the following observation about the relationship between this sponsored percentage and the transportation cost per unit $t$.

Observation 1: Given the price $\left\{p_{c}, p_{e}\right\}$ charged to end user and CSPs, if $0<n_{i}<1$, the sponsored content $\alpha_{i}^{*}(t)$ for CSP $i$ is a non-increasing function of $t$.

Observation 1 demonstrates that as the transportation cost per unit becomes smaller, the percentage of sponsored content increases (not strictly) due to competition. When the transportation cost per unit is small, the end users are more likely to choose the CSPs with higher user payoff level. The CSPs can use sponsored content strategy to pursue higher market share by improving the payoff of end users. This results in high percentage of sponsored content. An example for the observation 1, shown in Fig. 2, demonstrates the percentage of sponsored content under two cases: large CSP has higher user utility level, shown in Fig. 2(a) and lower user utility level, shown in Fig. 2(b). Under the first case, the small CSP has lower user utility level and revenue level. As the decrease of transportation cost per unit, the small CSP has to sponsor much higher percentage of content so as to remedy the huge disadvantages. Under the second case, the large CSP has to sponsor the content so as to take enough market shares while the small one sponsors no content due to its higher user utility level. As the decrease of transportation cost per unit, both the small and large CSPs have to sponsor much higher percentage of content so as to compete for more market shares. Even though the CSPs are likely to adopt high percentage of sponsored content with small transportation cost per unit, the market shares may not be improved. Another observation is taken about the relationship between the market share and transportation cost per unit. 


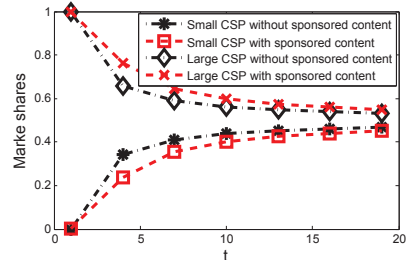

(a) Small CSP with higher user utility level $\left(\sigma_{e_{1}}=1, \sigma_{e_{2}}=1.5\right)$

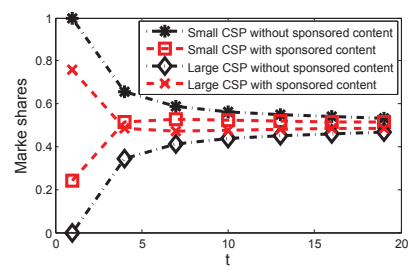

(b) Large CSP with higher user utility level $\left(\sigma_{e_{1}}=1.5, \sigma_{e_{2}}=1\right)$
Fig. 3: An example of market shares in the long run $\left(\sigma_{c_{1}}=\right.$ $1, \sigma_{c_{2}}=2, \beta=0.5, p_{e}=0.5, p_{c}=1$ )

Observation 2: Given prices $\left\{p_{c}, p_{e}\right\}$ charged to CSPs and end users, sponsored content strategy in the long run makes the market share of large CSP larger and small CSP smaller.

Obvervation 2 verifies the concerns of neutrality advocator that large CSP can always benefit more with the sponsored content strategy. When the utility level of the large CSP is high, its market share can be extended larger, compared with the small one, with sponsored content strategy. When the utility level is low, it can use sponsored content strategy to avoid the market share loss, or even obtain more market share than small one. An example shown in Fig. 3 is given to demonstrate these two kinds of scenarios with the same parameters with Fig. 2. Fig. 3(a) shows that as the transportation cost per unit becomes smaller, the small CSP with higher user utility level gets more market share. If the transportation cost per unit is small enough, it can even take the whole market. Yet, with the sponsored content strategy, the large CSP can improve the market share by providing higher sponsored content to its end users. This strategy remedies the disadvantage of its low user utility level. Fig. 3(b) demonstrates the case that the large CSP has higher utility level. With the sponsored content strategy, the market share gap between the large CSP and the small one becomes larger.

Summary. In the short run, the small CSP may benefit more from sponsored content. In the long run, the percentages of sponsored content for both CSPs are improved due to competition. The larger the transportation cost per unit, the higher percentage the CSPs sponsor their content. In addition, the large CSP can obtain more market shares by adopting sponsored content, verifying the concerns of network neutrality advocators.

\section{CONCLUSION}

In this paper, we study the impact of sponsored content strategy on the CSPs of different scales. We first analyze the interplay between CSPs, the monopolistic ISP and end users and conclude that none of CSPs have the incentive to sponsor their content if the monopolistic ISP can discriminate the price of each CSP. We then study the effect of competition by considering one small and one large CSP. We analyze the optimal strategies for both CSPs in the short-run competition and long-run competition. We conclude that the small CSP may benefit from the sponsored content strategy but not for large CSP in short-run competition. We also observe that in longrun competition, as the transportation cost per unit decreases, the optimal percentage of sponsored content increases. In addition, the market share of large CSP becomes larger with the sponsored content strategy. One direction of extending the current work is to consider the network with multiple ISPs. More detailed studies about the long-run competition are still necessary. We expect to work on these issues as future work.

\section{ACKNOWLEDGMENT}

Dan Wang's work is supported in part by National Natural Science Foundation of China (No. 61272464), RGC/GRF PolyU 5264/13E, HK PolyU 1-ZVC2, G-UB72.

\section{REFERENCES}

[1] Google, "International broadband pricing study: Dataset for public use." http://policybythenumbers.blogspot.gr/2012/08/internationalbroadband-pricing-study.html.

[2] H. Raj, S. Saroiu, A. Wolman, and J. Padhye, "Splitting the bill for mobile data with simlets," in Proc. ACM HotMobile, 2013.

[3] S. Ha, S. Sen, C. J. Wang, Y. Im, and M. Chiang, "Tube: Timedependent pricing for mobile data," in Proc. of ACM SIGCOMM, 2012.

[4] S. Sen, C. Joe-Wong, S. Ha, and M. Chiang, "Pricing data: A look at past proposals, current plans, and future trends," ACM Computing Surveys, vol. vol. V, no. No. N, 2013.

[5] P. Goldstein, "Google joins with india's bharti airtel for toll-free wireless internet service." http://www.fiercemobileit.com/story/att-ceo-contentproviders-asking-toll-free-data-plans/2012-07-18.

[6] R. Krause, "Senate may vet wheeler on 'toll-free' wireless data." http://news.investors.com/technology/053113-658386-wheelerfaces-net-neutrality-issues-at-confirmation.htm.

[7] S. Marek, "Verizon's shammo: Content providers see value in toll-free data model." http://www.fiercewireless.com/story/verizons-shammocontent-providers-see-value-toll-free-data-model/2013-05-22.

[8] J. Ankeny, "At\&t ceo: Content providers asking for 'toll-free' data plans." http://www.fiercemobileit.com/story/att-ceo-content-providersasking-toll-free-data-plans/2012-07-18.

[9] P. Goldstein, "Report: Espn in talks with u.s. carrier on toll-free data plans." http://www.fiercewireless.com/story/report-espn-talks-uscarrier-toll-free-data-plans/2013-05-10.

[10] R. T. Ma and V. Misra, "The public option: a non-regulatory alternative to network neutrality," in Proc. of ACM CoNEXT, 2011.

[11] P. Hande, M. Chiang, R. Calderbank, and S. Rangan, "Network pricing and rate allocation with content provider participation," in Proc. of IEEE INFOCOM, 2009.

[12] J. P. Choi and B.-C. Kim, "Net neutrality and investment incentives," in Rand Journal of Economics, 41(3):446-471, Autumn, 2010.

[13] M. Andrews, U. Ozen, M. I. Reiman, and Q. Wang, "Economic models of sponsored content in wireless networks with uncertain demand," in Proc. of Smart Data Pricing Workshop, IEEE, 2013.

[14] L. Zhang, W. Wu, and D. Wang, "Time-dependent pricing in wireless data networks: Flat-rate vs. usage-based schemes." To appear in Proc. of IEEE INFOCOM, 2014.

[15] L. Zhang, W. Wu, and D. Wang, "The effectiveness of controlling usage incentives in wireless data network." Poster, in Proc. of ACM SIGCOMM, 2013.

[16] A. Odlyzko, "The volume and value of information," International Journal of Communication, vol. vol. 6, 2012.

[17] C. Courcoubetis, K. Sdrolias, and R. Weber, "Revenue models, price differentiation and network neutrality implications in the internet," in Proc. of ACM NetEcon, 2013.

[18] L. Zhang and D. Wang, "Sponsoring content: Motivation and pitfalls for content service providers," tech. rep. Avialible at http://www4.comp.polyu.edu.hk/ cslizhang/InfocomW14-TR.pdf.

[19] R. Singel, "Youtubes bandwidth bill is zero. welcome to the new net." http://www.wired.com/business/2009/10/youtube-bandwidth/.

[20] A. Odlyzko, "Will smart pricing fnally take off?."

[21] M. J. Osborne and A. Rubinstein, "A course in game theory," MIT press, 1994. 